IFT-UAM/CSIC-00-01

hep-th/0001016

\title{
A Comment on the Holographic Renormalization Group and the Soft Dilaton Theorem
}

\author{
Enrique Álvarez † and César Gómez ษ \\ $\dagger$ Theory Division, CERN,1211 Geneva 23, Switzerland, \\ Instituto de Física Teórica, C-XVI, ] and Departamento de Física Teórica, C-XI, \\ Universidad Autónoma de Madrid E-28049-Madrid, Spain
}

\begin{abstract}
The equivalence between the holographic renormalization group and the soft dilaton theorem is shown for a class of warpped metrics solutions of the string beta function equations for the bosonic string.
\end{abstract}

\footnotetext{
${ }^{1}$ E-mail: enrique.alvarez, cesar.comez @uam.es

${ }^{2}$ Unidad de Investigación Asociada al Centro de Física Miguel Catalán (C.S.I.C.)
} 
A renormalization group interpretation of the holographic map ([15] [13] [23]) between quantum field theories (QFT) in four dimensions and classical gravity in five-dimensional space-time has been the subject of a bunch of recent papers ([2] [3] [4] [45] [6] [11] [12] [20] [8] [22]). The main idea underlying the renormalization group approach (RGA) to holography stems from the interpretation of the running of the coupling constants of the four dimensional QFT as Einstein's equations of five-dimensional gravity plus matter. This approach rests on the holographic map relating QFT couplings with boundary values of five-dimensional background fields.

Let $S\left(g_{\mu \nu}, \Phi_{i}\right)$ stand for the classical Einstein action evaluated on shell, at the points $g_{\mu \nu}(\bar{x}, \rho), \Phi_{i}(\bar{x}, \rho)$ solutions of the classical equations of motion with fixed boundary conditions, $g_{\mu \nu}(\bar{x}) \equiv g_{\mu \nu}(\bar{x}, \rho=\infty) ; \Phi_{i}(\bar{x}) \equiv \Phi_{i}(\bar{x}, \rho=\infty)$ at $\rho=\infty$. The space-time background metric preserving four dimensional Poincare invariance would be given by

$$
d s^{2}=g_{\mu \nu}(\bar{x}, \rho) d x^{\mu} d x^{\nu}+d \rho^{2}
$$

The holographic map (15] 132 [23) dictates that QFT correlators are given in the large $N$ limit in terms of the classical action $S\left(g_{\mu \nu}, \Phi_{i}\right)$ through:

$$
<\Theta_{i_{1}}\left(\bar{x}_{1}\right), \ldots \Theta_{i_{n}}\left(\bar{x}_{n}\right)>=\left.\frac{\delta^{n} S\left(g_{\mu \nu}, \Phi_{i}\right)}{\delta \Phi_{i_{1}}\left(x_{i_{1}}\right) \ldots \delta \Phi_{i_{n}}\left(x_{i_{n}}\right)}\right|_{\Phi_{i}=\Phi_{i}^{(0)}}
$$

where the $\Phi_{i}^{(0)}$ parametrize the particular QFT under consideration, and where the correspondence

$$
\Phi_{i} \rightarrow \Theta_{i}
$$

between five dimensional fields and four dimensional local observables has been employed.

The five dimensional bulk action $S\left(g_{\mu \nu}, \Phi_{i}\right)$ is infrared (IR) divergent (due to the contributions of the boundary $\rho=\infty$ ). This corresponds to QFT ultraviolet (UV) infinities, and is the essential ingredient of the IR/UV connection (21]).A regulator can be easily introduced, for example, by cutting down the region $\rho>\Lambda$ (cf. [14] [8] [22]), and be used to define, in Wilsonian sense, correlators at the scale $\Lambda$. Demanding that physical observables 
should be independent of the particular value chosen for $\Lambda$ then implements the CallanSymanzik (CS) equations of the corresponding QFT. From the five-dimensional bulk point of view, this is equivalent to demanding invariance under translations in the holographic direction, $\rho \rightarrow \rho+a$. In $([\mathbb{8}])$ the CS equation was thus recovered from the Hamilton constraint $H=0$ corresponding to the holographic time $\rho$ \&

Given a generic metric where four dimensions are fibered in a nontrivial way over the holographic coordinate, such as:

$$
d s^{2}=a^{2}(\rho) d \vec{x}_{4}^{2}+d \rho^{2}
$$

where $d \vec{x}_{4}^{2}$ stands for the flat minkowskian metric, the holographic version of Callan Symanzik equation for dimensionless physical quantities $A$, is ( [8]):

$$
\left(a \frac{\partial}{\partial a}-\beta_{i} \frac{\partial}{\partial \Phi_{i}}\right) A=0
$$

with

$$
\beta_{i} \equiv a \frac{\partial}{\partial a} \Phi_{i}
$$

It is then plain that the warping factor a plays precisely the rôle of the renormalization group scale $\mu$. [

The purpose of this letter is to relate equation (5) with the string soft dilaton theorem [1].

Let us consider the simplest case, namely the open bosonic string. Divergences of pure gluonic one-loop amplitudes come from the boundary of the moduli space of the annulus. It is a well known fact since the early days of string theory that those divergences could be absorbed into a renormalization of both the string coupling constant, $g$ and the string tension, $\alpha^{\prime}$. To be specific, the open string one loop ultraviolet divergences

\footnotetext{
${ }^{2}$ A Hamilton-Jacobi approach to the renormalization group for non critical strings on cosmological backgrounds was also pointed out by Polyakov in reference (16])

${ }^{3}$ For an early version of this identification, based on Polyakov's ideas for the confining string description of pure Yang-Mills (17] 18] [19), see 四.
} 
can be represented as insertions of closed string dilaton vertex operators evaluated at at zero momentum . This is the reason why the infinities can be absorbed into changes of $\alpha^{\prime}$, because those correspond to dilaton insertions. The full soft dilaton theorem can be summarized in the following equation ([1])

$$
\left(l_{s} \frac{\partial}{\partial l_{s}}-\frac{(d-2) g}{4} \frac{\partial}{\partial g}\right) A\left(\kappa_{1} \ldots \kappa_{n}\right)=\lim _{p \rightarrow 0} A\left(p ; \kappa_{1} \ldots \kappa_{n}\right) J \Delta(p) .
$$

where $\alpha^{\prime} \equiv l_{s}^{2}, d$ is the space-time dimension in which the gluonic momenta, $\kappa_{1} \ldots \kappa_{n}$ can lie, and in the right hand side $J$ is zero momentum dilaton tadpole and $\Delta(p)$ the dilaton propagator. The coupling $g$ is the open string coupling constant.

It is perhaps worth stressing that the validity of equation (77) [17] 18]does not depend on working in the critical dimension.

When the string background is a consistent solution of the sigma model conformal invariance conditions ([9], [10]) dilaton tadpoles should vanish, and the equation becomes:

$$
\left(l_{s} \frac{\partial}{\partial l_{s}}-\frac{(d-2) g}{4} \frac{\partial}{\partial g}\right) A\left(\kappa_{1} \ldots \kappa_{n}\right)=0,
$$

which when $d=4$ will be taken as our starting point. Let us evaluate it for a conformally invariant string background, i.e., to lowest order in $\alpha^{\prime}$, a solution of the equations:

$$
\begin{gathered}
R_{\mu \nu}-\nabla_{\mu} \nabla_{\nu} \Phi=0 \\
\nabla^{2} \Phi+(\nabla \Phi)^{2}=0
\end{gathered}
$$

Based on the soft dilaton theorem, the following solution was derived in ([5])

$$
\begin{array}{r}
d s^{2}=\frac{\rho}{l_{c}} d \vec{x}_{4}^{2}+d \rho^{2} \\
\Phi(\rho)=-\log \rho
\end{array}
$$

In [5] [6] has been argued that this metric is in some sense a universal string background canonically associated to gauge quantum field theories. Using now the specific dilaton dependence as determined in (10) in equation (6) yields:

$$
\beta_{\Phi}=a \frac{\partial \Phi}{\partial a}=-2
$$


which turns the holographic renormalization group equation (5) into:

$$
\left(a \frac{\partial}{\partial a}+2 \frac{\partial}{\partial \Phi}\right) A=0
$$

Using now the well-known relationship between the string coupling and the dilaton background namely,

$$
g \equiv e^{-\Phi / 2}
$$

and interpreting $\rho$ in the metric (10) as a four-dimensional running string tension ([19]) easily yields from (12)

$$
\left(l_{s} \frac{\partial}{\partial l_{s}}-\frac{(d-2) g}{4} \frac{\partial}{\partial g}\right) A=0
$$

which is exactly the four dimensional soft dilaton theorem.

Please note that from the present point of view the dynamics underlying the CallanSymanzik equation is just the conditions for the conformal invariance of the two-dimensional string sigma model and the fact that open string one loop divergences just happen to be represented by insertions of closed string vertex operators.

As a general comment, it is plain that the open/closed interelationship (as encoded in the soft dilaton theorem and otherwise) seems to underlie the whole holographic interpretation of gauge theories.

Four dimensional conformally invariant field theories, defined by $\beta_{i}=0$, do not renormalize the string coupling constant. Both this fact and the numerical value $(0)$ of the second member of the background equation f (9) are related to poorly understood (closed) tachyon condensates and the Liouville nature of the holographic coordinate. Finally we address the attention of the reader to the peculiar (from the renormalization group viewpoint) minus sign both in the holographic renormalization group (equation 5) and in the soft dilaton theorem equation (7). From the Hamilton-Jacobi point of view [8] this sign could be related with the wrong sign of the conformal factor in Einstein action while in the context of the soft dilaton theorem the sign is related to invariance of the gravitational

\footnotetext{
${ }^{4}$ Which in turn uniquely selects $d=4$.
} 
constant $\kappa$ with respect to shifts of the dilaton field. The sign in (14) depends on the particular identification between $\rho$ and $l_{s}$; we have used the dimension of $\rho$ as defined by the metric (10).

\section{Acknowledgments}

This work has been supported by the European Union TMR programs FMRX-CT960012 Integrability, Non-perturbative Effects, and Symmetry in Quantum Field Theory and ERBFMRX-CT96-0090 Beyond the Standard model as well as by the Spanish grants AEN96-1655 and AEN96-1664.

\section{References}

[1] M. Ademollo, A. D'Adda, R. D'Auria, F. Gliozzi, E. Napolitano, S. Sciuto and P. Di Vecchia, Soft Dilatons and Scale Renormalization in Dual Theories, Nucl. Phys. B94 (1975)221.

[2] E. T. Akhmedov, A remark on the AdS/CFT correspondence and the renormalization group flow, hep-th/9806217; Phys.Lett. B442 (1998) 152-15

[3] E. Álvarez and C. Gómez, Geometric Holography, the Renormalization Group and the c-Theorem, hep-th/9807226, Nucl.Phys. B541 (1999) 441-460.

[4] E. Álvarez and C. Gómez, Noncritical confining strings and the renormalization group, hep-th 9902012; Nucl. Phys. B550 (1999),169

[5] Enrique Álvarez and César Gómez, The Confining String from the Soft Dilaton Theorem, hep-th/9907158. 
[6] E. Álvarez and C. Gómez, The Renormalization Group Approach to the Confining String, hep-th/9911215

[7] V. Balasubramanian and P. Kraus, Spacetime and the Holographic Renormalization Group, hep-th/9903190.

[8] J. de Boer, E. Verlinde, H. Verlinde, On the Holographic Renormalization Group, hep-th/9912012

[9] C. Callan, E. Martinec, M. Perry and D. Friedan, Strings in Background Fields Nucl. Phys. B262 (1985),593.

[10] G. Curci and G. Paffuti, Consistency between the string background equations of motion and the vanishing of the conformal anomaly, Nucl. Phys. B286 (1987),399.

[11] D.Z. Freedman,SS Gubser, K. Pilch, N.P. Warner, Renormalization group flows from holographic supersymmetry and a c-theorem, hep-th/9906164.

[12] L Girardello,M. Petrini,M. Porrati, A. Zaffaroni, Novel local CFT and exact results on perturbations of $N=4$ super Yang Mills from AdS dynamics, hep-th/9810126.

[13] S.S. Gubser, I.R. Klebanov and A.M. Polyakov, Gauge theory correlators for noncritical string theory, hep-th/9802109.

[14] M. Henningson and K. Skenderis, The Holographic Weyl Anomaly hep-th/9806087.

[15] J. Maldacena, The Large N Limit of Superconformal Field Theories and Supergravity, hep-th/9711200.

[16] A.M. Polyakov, A few projects in string theory, Les Houches,1992.

[17] A.M. Polyakov, Confining Strings, hep-th/9607049.

[18] A. M. Polyakov, String Theory and Quark Confinement, hep-th/9711002. 
[19] A.M. Polyakov, The wall of the Cave, hep-th/9808057.

[20] M. Porrati, A. Starinets, $R G$ fixed points in supergravity duals of 4-d field theory and asymptotically AdS spaces, hep-th/9903241.

[21] L. Susskind and E. Witten, The Holomorphic Bound in AdS-Spaces, hep-th/9805114.

[22] E. Verlinde, H. Verlinde, RG-Flow, Gravity and the Cosmological Constant, hep-th/9912018;

[23] E. Witten, Anti de Sitter space and holography, hep-th/9802150. 